'Programa de formación de especialista en Radioterapia Oncológica. Escuela de Postgrado, Facultad de Medicina. Universidad de Chile. Santiago, Chile.

2Unidad de Braquiterapia, Servicio de Radioterapia, Instituto Nacional del Cáncer. Universidad San Sebastián. Santiago, Chile. ${ }^{a}$ Tecnólogo Médico. ${ }^{\text {bEnfermera. }}$

Este estudio no cuenta con apoyo financiero.

Recibido el 3 de octubre de 2018, aceptado el 30 de julio de 2019.

Correspondencia a: Apolo Salgado F. Avda. Profesor Zañartu 1010, Independencia. Santiago, Chile. asalgado@incancer.cl

\section{Image-Guided BrachyAblation (IGBA) en hepatocarcinoma. Descripción de la técnica y reporte del primer caso en Chile}

\author{
EVELYN SAN MARTÍN M. ${ }^{1}$, APOLO SALGADO F. ${ }^{2}$, \\ IGNACIO VÉLIZ R. ${ }^{\mathrm{a}}$, CYNTHIA VILLEGAS P. ${ }^{2, \mathrm{a}}$, BEATRIZ VÁSQUEZ F. ${ }^{2, \mathrm{~b}}$, \\ VINCKA PARRA C., , , JESSICA AROS B., ,b

\section{Image-guided brachyablation for hepatocarcinoma. Report of one case}

Eighty percent of hepatocarcinomas are inoperable at the moment of diagnosis. Liver transplantation is the treatment of choice in these cases, but local therapies are another alternative. Among these, Image-Guided BrachyAblation is a safe choice. We report a 76-year-old male with a hepatocarcinoma, who was considered inoperable due to the high surgical risk of the patient. A local treatment with Image-Guided BrachyAblation was decided. A brachytherapy needle was placed in the tumor under computed tomography guidance and a $15 \mathrm{~Gy}$ single dose was delivered from an Iridium-192 source. The patient had no immediate complications and at one month of follow up he continued without incidents.

(Rev Med Chile 2019; 147: 808-812)

Key words: Brachytherapy; Carcinoma, Hepatocellular; Radiotherapy, Image-Guided.

\section{A} nivel mundial, el cáncer hepático es el quinto cáncer más común y el tercero con mayor mortalidad, siendo el hepatocarcinoma (HCC) el tipo histológico más frecuente. La resección quirúrgica es considerada el tratamiento de primera línea ${ }^{1}$, con una sobrevida global de $40-70 \%$ a 5 años y recurrencia local a 5 años de $75 \%{ }^{1,2}$. El $80 \%$ de los HCC será irresecable al momento del diagnóstico, por cirrosis avanzada o comorbilidades ${ }^{3}$, siendo el trasplante hepático el tratamiento de elección ${ }^{1}$. Lamentablemente, existen limitantes para su realización, incluyendo las comorbilidades de los pacientes, inclumplimiento de los criterios de Milán, baja tasa de donantes y largos tiempos de espera, con la consiguiente progresión de enfermedad ${ }^{4}$. Es por ello que existen diversos tratamientos locales, incluyendo la ablación térmica (RFA), quimioembolización, radioterapia estereotáxica y braquiterapia de alta tasa (BT HDR) intersticial ${ }^{2,3,5-8}$, entre otros.

La Image-Guided BrachyAblation (IGBA) consiste en una BT HDR intersticial guiada por imágenes y es un tratamiento ablativo mínimamente invasivo que ha tenido resultados prometedores en tumores hepáticos primarios, secundarios e inclusive con tumores no susceptibles a RFA ${ }^{7,9-13}$. Las ventajas de esta técnica son no tener restricciones respecto al tamaño tumoral, ni estar limitada por estructuras termosensibles ${ }^{9,14}$, su efecto no varía por el movimiento del paciente o la excursión respiratoria, ya que el aplicador está inserto en el tumor $^{15}$, requiere instalaciones de fácil disponibilidad y es, comparativamente, de bajo costo ${ }^{11,12,16,17}$.

En Chile, al año 2012 se habían diagnosticado 1.066 tumores hepáticos nuevos ${ }^{18}$, de los cuales, cerca de 900 corresponderían a HCC. Si 80\% fuera 
irresecable, 720 pacientes hubieran sido posibles candidatos a trasplante hepático. En el año 2015 se registraron 52 trasplantes hepáticos ${ }^{19}$, por lo que si no ha existido mayor variación en la conducta, un gran número requirá otra estrategia de tratamiento.

Actualmente, el centro público oncológico Instituto Nacional del Cáncer (INC) cuenta con variados tipos de tratamientos, incluyendo la BT HDR intersticial con apoyo imagenológico, tanto de tomografía computada (TC) como de ecógrafo, pero no dispone de RFA, por lo que el propósito de este trabajo es describir el primer caso reportado de HCC tratado por medio de la técnica IGBA en Chile.

\section{Descripción del caso clínico}

Paciente de sexo masculino, de 76 años, con antecedentes de daño hepático crónico (DHC) por alcohol, hipertensión arterial, diabetes mellitus tipo 2, fibrilación auricular en anticoagulación oral, insuficiencia cardíaca crónica, accidente cerebrovascular secuelado con hemiparesia espástica izquierda y cáncer testicular tipo seminoma puro tratado en el año 2000 con quimioterapia. En seguimiento por cáncer testicular se realizó ecografía abdominal que mostró nódulo hepático de $26 \mathrm{~mm}$ sugerente de hepatocarcinoma. TC tórax, abdomen y pelvis identificó foco hipervascular hepático sugerente de HCC, asociado a DHC, sin otras anomalías y resonancia nuclear magnética (RNM) confirma que nódulo hepático hipervascular de $25 \mathrm{~mm}$ x $15 \mathrm{~mm}$ en aspecto anterior del segmento IV, compatible con HCC (LIRADS 5). No se observaron otras lesiones tumorales en el abdomen.

El paciente no tenía ascitis y no había signos de encefalopatía portal. La función hepática con leve hiperbilirrubinemia de $2,1 \mathrm{mg} / \mathrm{dL}$, fosfatasas alcalinas $339 \mathrm{U} / \mathrm{I}$; albuminemia 3,5 g/dL. La protrombinemia no fue considerada por terapia anticoagulante. El nivel de alfa fetoproteína (AFP) estaba levemente elevado $(10 \mathrm{ug} / \mathrm{L})$. Virus hepatitis $\mathrm{B}$ y $\mathrm{C}$ negativos.

Se discutió caso con equipo multidisciplinario, que por alto riesgo quirúrgico se catalogó como inoperable y se decidió tratamiento local con IGBA, considerando que no se cuenta con RFA, lo que correspondería a la técnica de elección.

\section{Descripción de la técnica y planificación de tratamiento}

1. El paciente se posiciona en decúbito supino con ambos brazos detrás de la cabeza en una camilla plana. Se conecta a monitoreo de signos vitales y se procede a instalación de vía periférica.

2. Se ubican fiduciales radiopacos sobre piel de paciente para marcaje de isocentro.

3. Se realiza barrido con TC de simulación céfalo-caudal, con toma de imágenes cada $5 \mathrm{~mm}$, para planificar inserción de dispositivo.

4. Luego de visualizar las imágenes obtenidas y delimitar la zona de inserción de agujas para IGBA con marcador permanente, se procede a la aplicación de anestesia local con lidocaína al $1 \%$ y sedación consciente endovenosa con midazolam $(1 \mathrm{mg})$ asociado a fentanilo (100 gammas).

5. Se limpia la zona bajo técnica estéril y se arma el campo quirúrgico. Posteriormente, se fija a piel el template sandwich HDR200 (MickNuclear System ${ }^{\circledR}$, Mount Vernon NY, USA) previamente armado y se inserta una aguja de $21 \mathrm{G}$ para marcaje de zona a tratar.

6. Se realiza nuevo TC simulación céfalo-caudal (cortes a $3 \mathrm{~mm}$ ), y confirmando la posición de aguja en sitio adecuado se procede a instalar aguja de BT de $17 \mathrm{G}$ y $20 \mathrm{~cm}$ de longitud en la zona a tratar, con la profundidad y angulación requerida para inserción intratumoral, que en este caso fue de $12 \mathrm{~cm}$, con una angulación de $15^{\circ}$ hacia lateral (Figura 1).

7. Se confirma posición adecuada de aguja por medio de TC simulación con contraste (cortes a $3 \mathrm{~mm}$ ) y se fija complejo template-aguja a piel.

8. Se traslada paciente a búnker de BT HDR con monitoreo de signos vitales continuo y se evalúan posibles complicaciones.

9. Con imágenes de TC simulación se realiza planificación de IGBA con marcaje de targets y órgano de riesgo (hígado sin tumor). Considerando óptima la dosimetría con prescripción al 100\% de la dosis (D100) de 15 Gray (Gy) en fracción única, y órgano de riesgo con dosis aceptable, se procede a tratamiento con fuente de ${ }^{192} \operatorname{Ir}$ (Figura 2 y 3 ).

10. El tratamiento es completado en aproximadamente $20 \mathrm{~min}$, y una vez finalizado se procede a retiro de implante con aseo posterior de la zona. 

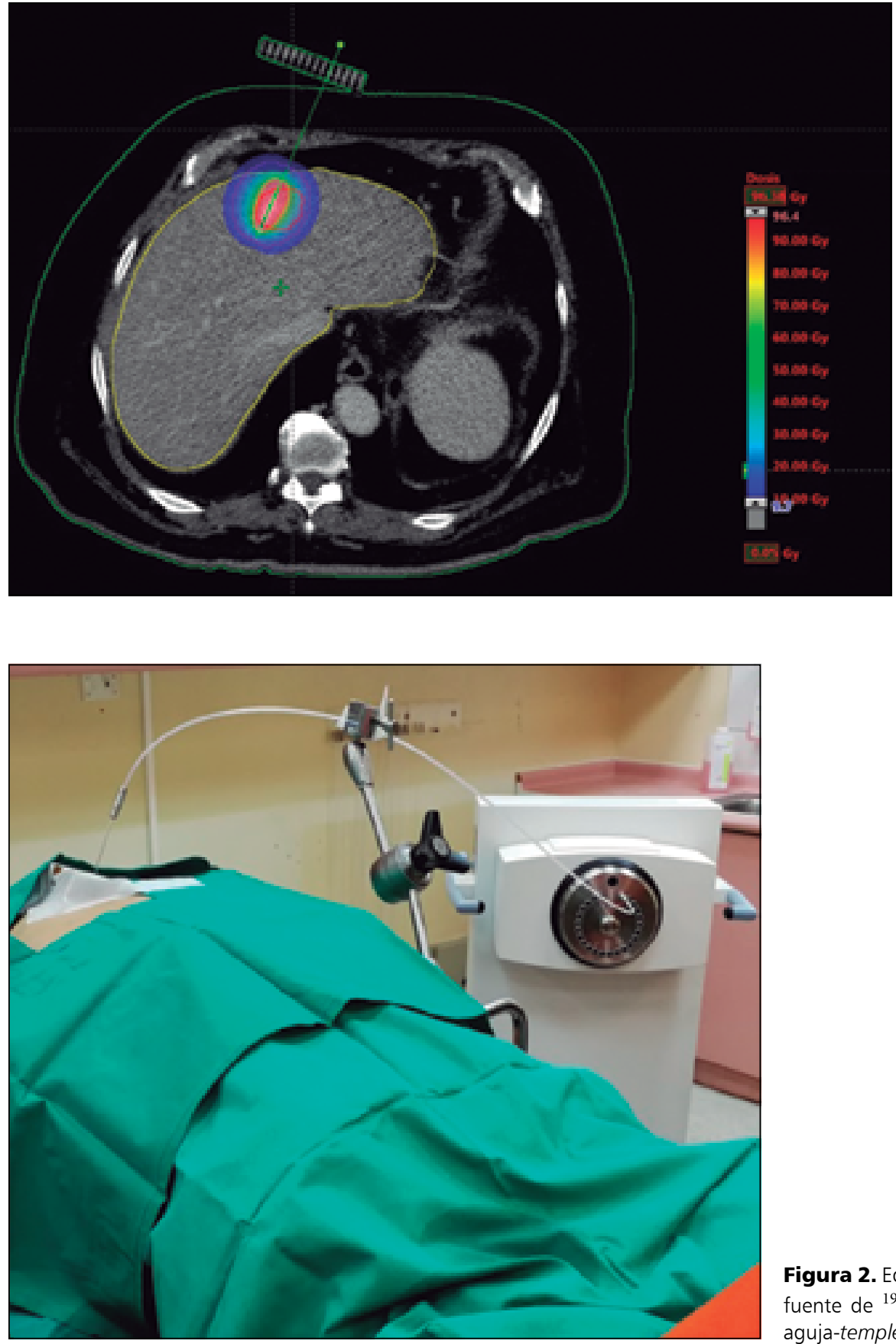
aguja-templete.
Figura 1. TC simulación con aguja de BT inserta intratumoral. El óvalo rojo representa el HCC y la curva morada a la isodosis de 15 Gy que lo envuelve.

Figura 2. Equipo de carga diferida con fuente de ${ }^{192}$ Ir conectado a complejo

11. Se deja a paciente hospitalizado para observación de posibles complicaciones, con hidratación endovenosa y profilaxis antibiótica.

12. El paciente completa un período de $24 \mathrm{~h}$ de observación.
En el caso de nuestro paciente no hubo incidentes. Fue dado de alta de acuerdo a lo programado y en el control de seguimiento al mes, se observó una reducción del tamaño de la lesión $(20 \mathrm{~mm}$ x $10 \mathrm{~mm}$ ). Se mantenía sin complicaciones. 


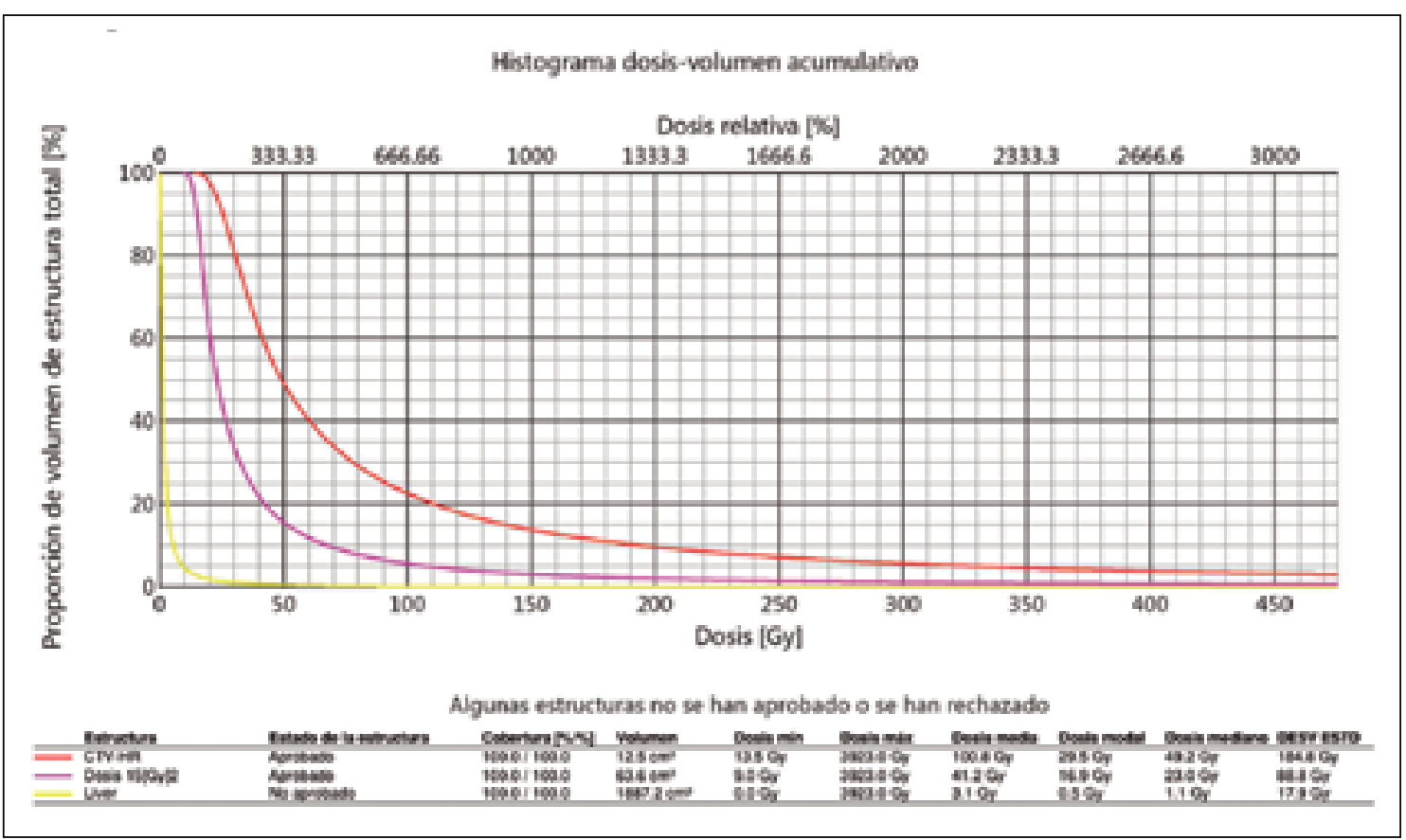

Figura 3. Histograma dosis-volumen.

\section{Discusión}

El tratamiento con IGBA en HCC se ha descrito como una terapia con gran efectividad en cuanto a control local, cercana a $90 \%$ al año ${ }^{14}$, que se logra por el efecto citotóxico, que puede ser potente durante semanas y meses, con el beneficio de entregar la dosis deseada al tumor con mínima exposición a órganos circundantes debido a la alta gradiente de dosis característica de la fuente de ${ }^{192} \operatorname{Ir}^{20}$.

Esta técnica es similar a la utilizada en la RFA, utilizando la inserción de aguja de forma percutánea hacia el sitio del tumor ${ }^{3}$. Puede ser utilizada en la mayoría de las regiones corporales ${ }^{5}$, teniendo cuidado de la protección de los órganos de riesgo ${ }^{13}$ y las complicaciones asociadas posibles, las cuales son anecdóticas y generalmente de manejo conservador (sangrado, infección, abscesos y ulceraciones $)^{17}$.

El presente reporte de caso demuestra la factibilidad de la realización de IGBA en Chile, en centros que cuenten con el equipo técnico y con profesionales capacitados para su cumplimiento con estándares de calidad necesarios. Conside- rando los recursos limitados del sistema de salud chileno, debiese considerarse esta técnica como una opción para pacientes con HCC irresecable y que no sean candidatos al tratamiento estándar, dada su facilidad de implementación al no requerir personal de difícil entrenamiento, además de ser una técnica con buen perfil de seguridad y excelente costo-efectividad.

Agradecimientos: Técnicos paramédicos de la Unidad de Braquiterapia, Servicio de Radioterapia, Instituto Nacional del Cáncer. Santiago, Chile. Auxiliares de la Unidad de Braquiterapia, Servicio de Radioterapia, Instituto Nacional del Cáncer. Santiago, Chile.

\section{Referencias}

1. Forner A, Reig M, Bruix J. Hepatocellular carcinoma. Lancet 2018; 391 (10127): 1301-14.

2. Tabrizian P, Roayaie S, Schwartz ME. Current management of hepatocellular carcinoma. World J Gastroenterol 2014; 20 (30): 10223-37.

3. Mohnike K, Wieners G, Pech M, Seidensticker M, Rühl 
R, López-Haenninen E, et al. Image-Guided Interstitial High-Dose-Rate Brachytherapy in Hepatocellular Carcinoma. DDI 2009; 27 (2): 170-4.

4. Freeman RB, Steffick DE, Guidinger MK, Farmer DG, Berg CL, Merion RM. Liver and Intestine Transplantation in the United States, 1997-2006. Am J Transplan 2008; 8 (4p2): 958-76.

5. Bretschneider T, Ricke J, Gebauer B, Streitparth F. Image-guided high-dose-rate brachytherapy of malignancies in various inner organs - technique, indications, and perspectives. J Contemp Brachytherapy 2016; 8 (3): 253-63.

6. Hass P, Mohnike K. Extending the Frontiers Beyond Thermal Ablation by Radiofrequency Ablation: SBRT, Brachytherapy, SIRT (Radioembolization). Viszeralmedizin 2014; 30 (4): 3-3.

7. Collettini F, Schreiber N, Schnapauff D, Denecke T, Wust P, Schott E, et al. CT-guided high-dose-rate brachytherapy of unresectable hepatocellular carcinoma. Strahlenther Onkol 2015; 191 (5): 405-12.

8. Doi H, Beppu N, Kitajima K, Kuribayashi K. Stereotactic Body Radiation Therapy for Liver Tumors: Current Status and Perspectives. Anticancer Res 2018; 38 (2): 591-9.

9. Collettini F, Singh A, Schnapauff D, Powerski MJ, Denecke T, Wust $\mathrm{P}$, et al. Computed-tomography-guided high-dose-rate brachytherapy (CT-HDRBT) ablation of metastases adjacent to the liver hilum. Eur J Radiol 2013; 82 (10): e509-14.

10. Ricke J, Mohnike K, Pech M, Seidensticker M, Rühl R, Wieners $\mathrm{G}$, et al. Local response and impact on survival after local ablation of liver metastases from colorectal carcinoma by computed tomography-guided high-dose-rate brachytherapy. Int J Radiat Oncol Biol Phys 2010; 78 (2): 479-85.

11. Ricke J, Wust P, Wieners G, Beck A, Cho CH, Seidensticker $\mathrm{M}$, et al. Liver malignancies: CT-guided interstitial brachytherapy in patients with unfavorable lesions for thermal ablation. J Vasc Interv Radiol 2004; 15 (11): 1279-86.

12. Ricke J, Wust P, Stohlmann A, Beck A, Cho CH, Pech
$\mathrm{M}$, et al. CT-guided interstitial brachytherapy of liver malignancies alone or in combination with thermal ablation: phase I-II results of a novel technique. Int J Radiat Oncol Biol Phys 2004; 58 (5): 1496-505.

13. Ricke J, Wust P. Computed tomography-guided brachytherapy for liver cancer. Semin Radiat Oncol 2011; 21 (4): 287-93.

14. Collettini F, Schnapauff D, Poellinger A, Denecke T, Schott E, Berg T, et al. Hepatocellular carcinoma: computed-tomography-guided high-dose-rate brachytherapy (CT-HDRBT) ablation of large $(5-7 \mathrm{~cm})$ and very large $(>7 \mathrm{~cm})$ tumours. Eur Radiol 2012; 22 (5): 1101-9.

15. McGinn CJ, Ten Haken RK, Ensminger WD, Walker S, Wang S, Lawrence TS. Treatment of intrahepatic cancers with radiation doses based on a normal tissue complication probability model. J Clin Oncol 1998; 16 (6): 2246-52.

16. 231 CT-Guided brachytherapy of hepatocellular carcinoma in liver cirrhosis -A novel therapeutic approach. J Hepatol 2005; 42: 89.

17. Ricke J, Wust P, Wieners G, Hengst S, Pech M, López Hänninen E, et al. CT-guided interstitial single-fraction brachytherapy of lung tumors: phase I results of a novel technique. Chest 2005; 127 (6): 2237-42.

18. GLOBOCAN 2012 [Internet]. [citado el 10 de septiembre de 2018]. Disponible en: http://globocan.iarc. fr/old/summary_table_pop_prev.asp?selection $=38152$ \&title $=$ Chile \&sex $=0$ \&window $=1$ \&sort $=0$ \&submit $=\%$ C2\%A0Execute\%C2\%A0.

19. Informe de Procuramiento y Trasplante - 2015 - Sociedad Chilena de Trasplantes [Internet]. [citado el 10 de septiembre de 2018]. Disponible en: https://www. sociedaddetrasplante.cl/biblioteca/informe-de-procuramiento-y-trasplante/category/39-2015.html.

20. Lüdemann L, Wybranski C, Seidensticker M, Mohnike $\mathrm{K}$, Kropf S, Wust $\mathrm{P}$, et al. In vivo assessment of catheter positioning accuracy and prolonged irradiation time on liver tolerance dose after single-fraction 192Ir high-dose-rate brachytherapy. Radiat Oncol 2011; 6: 107. 